\title{
Similarity based TOPSIS with linguistic-quantifier based aggregation using OWA
}

\author{
Pasi Luukka \\ School of Business and Management, LUT University, \\ Yliopistonkatu 34, 53851 Lappeenranta, Finland \\ Email: pasi.luukka@lut.fi
}

\author{
Jan Stoklasa \\ School of Business and Management, LUT University \\ Yliopistonkatu 34, 53851 Lappeenranta, Finland, and \\ Palacky University Olomouc, Faculty of Arts \\ Department of Economic and Managerial Studies \\ Email: jan.stoklasa@lut.fi \\ Email: jan.stoklasa@upol.cz
}

\begin{abstract}
In this paper we present similarity based TOPSIS with OWA operators. The motivation behind this new method is the fact that in many real world problems it is more important to consider the amount of criteria that a particular alternative is able to satisfy instead of simply concentrating on the importance of particular criteria. Here with OWA operators we can tackle this problem together with multi-criteria decision making method called TOPSIS by aggregating alternatives' similarities towards positive ideal solution and negative ideal solution and aggregating these similarities using OWA. The use of linguistic quantifiers represented by OWA weights generated by a selected RIM quantifier allows for the reflection of decision-maker's attitude to risk in the calculation of the similarities of the alternative with positive and negative ideal solutions.
\end{abstract}

\section{INTRODUCTION}

$\mathbf{T}$ HE name TOPSIS is shortening from the Technique for Order Preference by Similarity to Ideal Solution. This tool belongs to multi-criteria decision making methods which are of increasing importance [1], [2]. TOPSIS is based on the idea of forming two ideal solutions (best possible case called the (positive) ideal solution and denoted PIS and worst possible case called the negative ideal solution and denoted NIS), both relative to the set of available alternatives, and comparing the current alternative to these two. Unlike the name of the method suggests originally [3] this was done by computing the distances of each alternative to both ideal solutions and then forming the so called relative closeness to the ideal solution from these distances. The relative closeness to the ideal solution is originally defined in [3] in such a way that its value is equal to 1 for alternatives identical with PIS and 0 for alternatives identical with NIS. This way the relative closeness to the ideal solution takes into account the minimization of the distance of an alternative from PIS and the maximization of its distance from NIS and introduces a specific tradeoff between the two distances. The distances from PIS and NIS are calculated as Euclidean distances and as such do not reflect any behavioral or personality traits of the decision-maker. The weights of criteria are already reflected

The research was supported by the Finnish Strategic Research Council, grant number 313396 / MFG40 - Manufacturing 4.0, and by LUT research platform AMBI- Analytics-based management for business and manufacturing industry. in the vectors representing all the alternatives, PIS and NIS in the calculation of relative closeness to the ideal solution.

In similarity based TOPSIS [4] similarity is used to compare the alternatives with both ideal solutions. Later a generalized version of the similarity based TOPSIS was developed [5], where the aggregation of similarities was done using Bonferroni mean [6].

TOPSIS has not been examined much in connection with OWA operators [7] and to our knowledge similarity based TOPSIS variants with OWA aggregation do not exist in earlier literature. Chen et al. [8] examined OWA operator together with standard TOPSIS and used OWA in both internal and external aggregation. Wang et al. [9] developed OWA-TOPSIS approach in intuitionistic fuzzy environment. There OWA was used to aggregate preference and source and to calculate the distance; overall six different types of information aggregation processes are analysed in the paper. Liu et al. [10] used OWA operators to create additive reciprocal matrices to be used as ideal solutions for TOPSIS. Also Yusoff et al.[11] applied Minkowski OWA distance to aggregate distances to positive and negative ideal solutions. However none of these OWA TOPSIS combinations consider aggregating the information on differences of values representing the alternatives under separate criteria into an overall distance or similarity with respect to positive and negative ideal solutions separately by posing different (possibly linguistic) requirements for the distance from or similarity to PIS and NIS.

Intuitively approaching the distance/similarity to PIS and NIS in a different way seems reasonable since if we want to pose a requirement as 'most' of the criteria should have highly similar values for the positive ideal solution and the alternative in question for the alternative to be considered similar to PIS or to be desirable, it is highly unlikely that we want to do the same with this alternative and negative ideal solution and still call it desirable; on the other hand we might require only 'a few' criteria having highly similar values for the NIS and the alternative in question to consider the alternative similar to NIS. Also notable is that if you apply same linguistic weights derived from OWA to aggregate both distances (to PIS and NIS) eventhough relative closeness values differ actual rankings usually does not show statistically 
significant differences [8].

Here we introduce similarity based TOPSIS with OWA operators with two different motivations behind this. One motivation is that with OWA operator we are able to make linguistic quantifications like 'at most half' 'almost all' or 'at least two'. All this can be done without expressing preference on which of the criteria are required to satisfy these needs. Besides this it is unlikely that we want to have high similarity on e.g. 'most' criteria to be met for NIS even though for PIS this would clearly be desirable. One could, for example, expect, that a careful decision-maker would require 'at least a few' high similarities with NIS across all the criteria to consider the alternative in question similar to NIS, but the same decision-maker would require 'most' of the values of criteria to be highly similar with PIS for the alternative to be considered similar to PIS. On the other hand a overly optimistic (i.e. less careful or more risk-taking) decision maker might consider 'a few' highly similar values of criteria between the alternative in question and PIS to be sufficient to consider it similar to $P I S$, while he/she would require 'almost all' the criteria to have similar values to those of NIS to consider the alternative in question as similar to NIS. Other linguistic quantifications that define an alternative similar to PIS and one similar to NIS can be also considered depending on the purpose of the model, the problem being solved and also on the characteristics, preferences and risk attitude of the decisionmaker. Customizability in this matter is definitely reasonable and can lead to better fitting decision support using TOPSIS. Hence in this paper we introduce two sets of linguistic weights for OWA separately for PIS and NIS. In context of supplier evaluation this kind of requirement is at least as important as simple weighting of criterions of their importance.

\section{Preliminaries}

Yager [7] defined ordered weighted averaging (OWA) operator as follows.

Definition 1: An ordered weighted averaging (OWA) operator of dimension $n$ is a mapping $F: \mathbb{R}^{n} \longrightarrow \mathbb{R}$, that has an associated weighting vector such that $w_{i} \in[0,1], 1 \leq i \leq n$, $\sum_{i=1}^{n} w_{i}=1$

$$
F\left(a_{1}, \ldots, a_{n}\right)=\sum_{j=1}^{n} w_{j} b_{j}=w_{1} b_{1}+\ldots+w_{n} b_{n},
$$

where $b_{j}$ is the $j$-th largest element of the collection of objects $a_{1}, a_{2}, \ldots, a_{n}$.

In our research we are interested in linguistic quantification of weights, or to be more precise of the quantification of the amount of criteria that need to be fulfilled/satisfied sufficiently. With linguistic quantification we mean terms like 'at least some' of the criteria, 'almost all' criteria etc. For this purpose quantifier guided aggregation with OWA operators was established in [12], [13]. One field of quantifiers is called RIM quantifier [12] which is defined as follows.

Definition 2: A fuzzy subset $\mathrm{Q}$ of the unit interval is called a Regular Increasing Monotone (RIM) quantifier, if it satisfies the following conditions

1) $Q(0)=0$,

2) $Q(1)=1$

3) $Q(x) \geq Q(y)$, if $x>y$.

The RIM quantifiers can be used to express terms like 'all', 'most', 'many' and 'at least $k$ ', where $k$ is an integer number. Often used quantifier is $Q(x)=x^{\alpha}, \alpha \geq 0$ where the weights are calculated as follows

$$
w_{i}=Q\left(\frac{i}{n}\right)-Q\left(\frac{i-1}{n}\right), \quad i=1, \ldots, n .
$$

By using this with proper selection of $\alpha$ we are able to model different types of linguistic terms as described in Table I which is following Yager's example.

Note that the linguistic quantifiers listed in Table I and represented by vectors of OWA weights in fact all have the 'at least' interpretation, in other words we are defining the quantifiers 'at least one', 'at least few', 'at least some', 'at least many', 'at least most' and 'at least all' in Table I. Even though Yager does not directly specify so in [7]. The reason for this might be that the linguistic labels without the 'at least' part are easier to understand and thus to be used by decisionmakers, and also for example 'at least all' is identical with 'all'. We, however, consider it important to point out that the linguistic quantifiers defined in Table I do not represent 'just one', 'just few', 'just some' and so on. We need to stress that the use of these quantifiers (and the respective OWA weight vectors) does not guarantee that only the specified amount of criteria will be satisfied sufficiently. It is possible that, for example, all the criteria will be satisfied to a high degree even if we use the 'few' quantifier. We therefore strongly suggest to keep the 'at least ...' meaning of the quantifiers in mind when using them.

TABLE I

WEIGHTS WITH DIFFERENT LINGUISTIC QUANTIFIERS

\begin{tabular}{|c|c|c|c|c|c|c|}
\hline Weight & At least one & Few & Some & Many & Most & All \\
\hline$\alpha$ & $\alpha \rightarrow 0$ & 0.1 & 0.5 & 2 & 10 & $\alpha \rightarrow \infty$ \\
\hline$w_{1}$ & 1 & 0.8513 & 0.4472 & 0.04 & 0 & 0 \\
\hline$w_{2}$ & 0 & 0.0611 & 0.1852 & 0.12 & 0.0001 & 0 \\
\hline$w_{3}$ & 0 & 0.0378 & 0.1421 & 0.20 & 0.0059 & 0 \\
\hline$w_{4}$ & 0 & 0.0277 & 0.1198 & 0.28 & 0.1013 & 0 \\
\hline$w_{5}$ & 0 & 0.0221 & 0.1056 & 0.36 & 0.8926 & 1 \\
\hline
\end{tabular}

\section{Method}

To apply similarity based TOPSIS with OWA operator we require a specification of the decision matrix for a set of alternatives over a set of criteria. Given a set of $m$ alternatives $A=\left\{a_{i} \mid i=1,2, \ldots, m\right\}$, a set of $n$ criteria $C=\left\{c_{j} \mid j=\right.$ $1,2, \ldots, n\}$ and a set of weigths $W=\left\{w_{j} \mid j=1,2, \ldots, n\right\}$, $w_{j}>0, \sum_{j=1}^{n} w_{j}=1$, where $w_{j}$ denotes the weight of the criterion $c_{j}$, let $X=\left\{x_{i j} \mid i=1,2, \ldots, m, j=1,2, \ldots, n\right\}$ denote the decision matrix where $x_{i j}$ is the performance measure of the alternative $a_{i}$ with respect to the criterion $c_{j}$. Besides this we will also be using two sets of OWA 
operator weights $W^{+}=\left\{w_{j}^{+} \mid j=1,2, \ldots, n\right\}$ used in the context of the similarity of an alternative to PIS and $W^{-}=\left\{w_{j}^{-} \mid j=1,2, \ldots, n\right\}$ used in the context of the similarity of an alternative to NIS. Given the decision matrix, the similarity based TOPSIS with OWA involves following steps.

1. Normalize the decision matrix into a unit interval.

$$
\begin{aligned}
& z_{i j}=\frac{x_{i j}-\min _{i}\left(x_{i j}\right)}{\max _{i}\left(x_{i j}\right)-\min _{i}\left(x_{i j}\right)}, \\
& i=1,2, \cdots, m, j=1,2, \cdots, n
\end{aligned}
$$

2. Compute the weighted normalized decision matrix $V=\left[v_{i j}\right]$ :

$$
v_{i j}=z_{i j} w_{j}, i=1, \cdots, m, j=1, \cdots, n
$$

3. Determine the positive and negative ideal solutions

$$
\begin{aligned}
& V^{+}=\left[v_{1}^{+}, \ldots, v_{m}^{+}\right] \text {such that } \\
& \qquad v_{j}^{+}=\left\{\begin{array}{l}
\max _{i} v_{i j} \text { if } j \in B \\
\min _{i} v_{i j} \text { if } j \in C
\end{array}\right.
\end{aligned}
$$

$$
\begin{aligned}
& V^{-}=\left[v_{1}^{-}, \ldots, v_{m}^{-}\right] \text {such that } \\
& \qquad v_{j}^{-}=\left\{\begin{array}{l}
\min _{i} v_{i j} \text { if } j \in B \\
\max _{i} v_{i j} \text { if } j \in C
\end{array}\right.
\end{aligned}
$$

where $B$ is the set of indices of benefit-type criteria, and $\mathrm{C}$ is the set of indices of cost-type criteria. Note that given the normalization (3) the definitions of $V^{+}$and $V^{-}$can be simplified, as we know that for any $j=1, \ldots, n$ it holds that:

$$
\begin{aligned}
& -\max _{i} v_{i j}=1 \cdot w_{j} \\
& -\min _{i} v_{i j}=0
\end{aligned}
$$

This means that it is sufficient to know the orientation of the criteria (cost/benefit type) in order to be able to define the PIS and NIS in this alternative of TOPSIS. For example if we consider five criteria $c_{1}, \ldots, c_{5}$ such that $1,2,5 \in B$ and $3,4 \in C$ then under (3) we automatically get $V^{+}=\left[w_{1}, w_{2}, 0,0, w_{5}\right]$ and $V^{-}=\left[0,0, w_{3}, w_{4}, 0\right]$ regardless of the actual performance values of the alternatives.

4. Compute OWA operator weights by using suitable quantifier for the linguistic requirement of aggregation. Since linguistic requirement for similarity toward positive ideal solution is clearly different than to negative ideal solution we can derive two sets of weights with different linguistic requirements for an alternative to be considered similar to PIS or to NIS.

$$
w_{j}^{+}=Q_{1}\left(\frac{j}{n}\right)-Q_{1}\left(\frac{j-1}{n}\right), \quad j=1, \cdots, n
$$

$$
w_{j}^{-}=Q_{2}\left(\frac{j}{n}\right)-Q_{2}\left(\frac{j-1}{n}\right), \quad j=1, \cdots, n
$$

where $Q_{1}$ and $Q_{2}$ denotes RIM functions for different linguistic requirements.

5. Compute similarity vector for each alternative $a_{i}$ w.r.t. positive ideal solution (i.e. the vectors $\left[s_{i 1}^{+}, \ldots, s_{i n}^{+}\right]$, $i=1, \ldots, m$ ) and negative ideal solution (i.e. the vectors $\left.\left[s_{i 1}^{-}, \ldots, s_{i n}^{-}\right], i=1, \ldots, m\right)$ :

$s_{i j}^{+}=\sqrt[p]{1-\left|\left(v_{i j}\right)^{p}-\left(v_{j}^{+}\right)^{p}\right|}, i=1, \cdots, m, j=1, \cdots, n$

$s_{i j}^{-}=\sqrt[p]{1-\left|\left(v_{i j}\right)^{p}-\left(v_{j}^{-}\right)^{p}\right|}, i=1, \cdots, m, j=1, \cdots, n$

Here $\hat{s}_{i}^{+}=\left[s_{i 1}^{+}, s_{i 2}^{+}, \cdots, s_{i n}^{+}\right]$denotes the similarity vector of the alternative $a_{i}$ with the positive ideal solution and $\hat{s}_{i}^{-}=\left[s_{i 1}^{-}, s_{i 2}^{-}, \cdots, s_{i n}^{-}\right]$denotes the similarity vector of the alternative $a_{i}$ with the negative ideal solution.

Theorem 1: Under the normalization (3), if $p=1$ then

$s_{i j}^{+}+s_{i j}^{-}=2-w_{j}$ for any $i=1, \ldots, m$ and $j=1, \ldots, n$

Proof 1: Since (3) normalizes the values in the decisionmatrix into a unit interval, either $v_{j}^{+}=1 \cdot w_{j} \wedge v_{j}^{-}=0$ or $v_{j}^{+}=0 \wedge v_{j}^{-}=1 \cdot w_{j}$ for any $j=1, \ldots, n$. Either way we get

$$
\begin{aligned}
s_{i j}^{+}+s_{i j}^{-}=1 & -\left|v_{i j}-0\right|+1-\left|v_{i j}-w_{j}\right|= \\
& =1-v_{i j}+1-\left(w_{j}-v_{i j}\right)=2-w_{j},
\end{aligned}
$$

because $v_{i, j} \in\left[0, w_{j}\right]$ for any $i=1, \ldots, m$ and $j=$ $1, \ldots, n$.

6. Compute the similarity of each alternative w.r.t. positive ideal solution and negative ideal solution by aggregating the respective similarity vector using the OWA operator. This aggregation can reflect the requirements on how many of the criteria need to have high similarity for the alternative and PIS (or NIS) for the alternative to be considered 'similar to PIS' (or 'similar to NIS'). These requirements can be expressed using the linguistic quantifiers summarized in Table I.

$$
s_{i}^{+}=\sum_{j=1}^{n} w_{j}^{+} b_{j}^{+}
$$

where $b_{j}^{+}$is the $j^{\text {th }}$ largest element of $\hat{s}_{i}^{+}=$ $\left[s_{i 1}^{+}, s_{i 2}^{+}, \cdots, s_{i n}^{+}\right]$.

Similarly

$$
s_{i}^{-}=\sum_{j=1}^{n} w_{j}^{-} b_{j}^{-}
$$

where $b_{j}^{-}$is the $j^{t h}$ largest element of $\hat{s}_{i}^{-}=$ $\left[s_{i 1}^{-}, s_{i 2}^{-}, \cdots, s_{i n}^{-}\right]$. 
Note that it is the $W^{+}$OWA weights that reflect the requirements for the alternative to be considered similar to PIS in terms of the linguistically quantified (described) minimum number of criteria with respect to which the alternative needs to be similar with PIS for the alternative to be considered 'similar to PIS overall'. On the other hand the $W^{-}$OWA weights reflect the requirements for the alternative to be considered similar to NIS in terms of the linguistically quantified (described) minimum number of criteria with respect to which the alternative needs to be similar with NIS for it to be considered similar to NIS overall'.

7. Compute the relative closeness of the alternative to the positive ideal solution:

$$
R C_{i}=\frac{s_{i}^{+}}{s_{i}^{+}+s_{i}^{-}}, i=1, \cdots, n
$$

The definition of $R C_{i}$ by (14) does not guarantee that full similarity with PIS $\left(s_{i}^{+}=1\right)$ would imply that $R C_{i}=1$ by itself. Also full similarity with NIS $\left(s_{i}^{-}=1\right)$ does not mean that $R C_{i}=0$. However, zero similarity with NIS $\left(s_{i}^{-}=0\right)$ does imply that $R C_{i}=1$ regardless of the actual similarity with PIS. Still increasing the similarity with PIS $\left(s_{i}^{+}\right)$increases $R C_{i}$ while increasing similarity with NIS $\left(s_{i}^{-}\right)$decreases the value of $R C_{i}$. It can therefore be considered a reasonable value for the ranking of alternatives.

8. Arrange the ranking indexes in a descending order with respect to the values of $R C_{i}$ to obtain the best alternative.

The above proposed method differs from original TOPSIS in four ways:

1) The normalization is done to unit interval unlike in the original version of TOPSIS. This simplifies the definition of PIS and NIS and makes it independent on the actual performance of the alternatives w.r.t. the criteria. It is sufficient to know the orientation of the criteria to be able to define PIS and NIS ${ }^{1}$.

2) The computation of how similar alternatives and ideal vectors (PIS and NIS) are is done using a similarity measure instead of a distance measure. This fully introduces the concept of similarity into a method that has a 'similarity to ideal solution' in its very name.

3) The aggregation of similarity vectors (criteria-wise similarities to PIS and NIS) is done using two different ordered weighted averaging operators. This allows different linguistic quantifications of "how similar an alternative needs to be to PIS or NIS criteria-wise to be considered 'overall similar to PIS or NIS' respectively". This opens doors for the reflection of the risk-preference of the decision-maker and for more detailed specification of the requirements on a 'alternative similar to PIS' and an 'alternative similar to NIS' by the decision-maker.

\footnotetext{
${ }^{1}$ In original method normalization is done as $z_{i j}=\frac{x_{i j}}{\sqrt{\sum_{i=1}^{n} x_{i j}^{2}}}, i=$ $1,2, \ldots, n, j=1,2, \ldots, m$.
}

The proposed method uses linguistic quantification for this purpose to facilitate the expression of these requirements for a wide set of decision-makers.

4) The relative closeness computation is adjusted to similarity measures instead of the distance measures that are used in the calculation of the relative closeness to the ideal solution in [3].

In the following example we will illustrate the performance of the method proposed in this paper on a supplier evaluation and selection problem. We will also show how different requirements on the similarity to PIS and NIS (potentially representing the risk-attitude of the decision-maker) can be reflected, and discuss how this can influence the results obtained by the method.

\section{SUPPLIER EVALUATION}

Here we introduce how we can use similarity based TOPSIS with OWA operators in supplier evaluation. Our basic problem is the following. A car manufacturing company wants to select its supplier. Most important criteria which manufacturer selected to focus on are: price, duration of the project, quality, the amount of equipment and distance. From these quality and the amount of equipment are considered to be benefit-type criteria and others are considered to be cost-type criteria. After preliminary selection, five suppliers remain and the decision matrix given in Table II is obtained.

Linguistic assessments for quality and equipment are transformed into numerical scale between $[0,10]$ resulting in Table III.

The first step is to calculate the normalized decision matrix which can be found in Table IV.

In this problem we consider all the criteria to be equally important leading to the weighting expressed by $W=[1, \ldots, 1]$ to be redundant. Even though the original method requires

TABLE II

ORIGINAL DECISION MATRIX

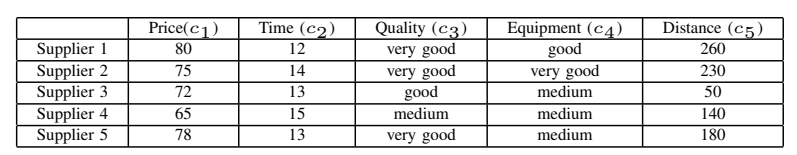

TABLE III

DECISION MATRIX ON NUMERICAL SCALE

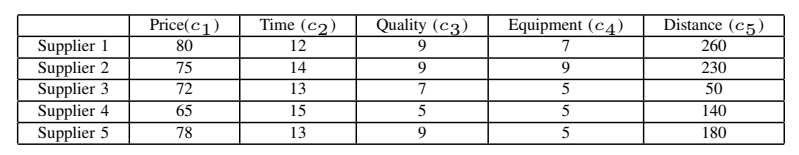

TABLE IV

NORMALIZED DECISION MATRIX

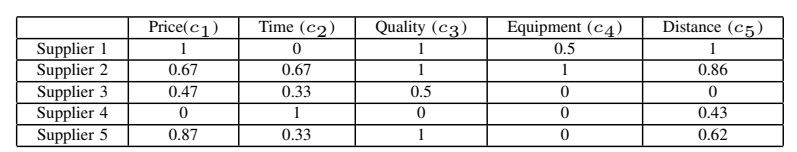


normalized weights, the final ordering of the alternatives (suppliers in this example) will not change if we do not normalize the weights and with the weighting vector $W=[1,1,1,1,1]$ the calculations will be easier to follow and will thus serve better as an example of the proposed method. This does not limit the applicability of the results presented further. It allows us to see the effects of linguistic quantification in the method more clearly. Given the fact that $c_{1}, c_{2}$ and $c_{5}$ are cost-type criteria and $c_{3}$ and $c_{4}$ are benefit-type criteria, we get the positive ideal solution in the form of $V^{+}=[0,0,1,1,0]$ and the negative ideal solution if the form of $V^{-}=[1,1,0,0,1]$.

The similarity vectors of the alternatives to PIS (vectors $\left.\hat{s}_{i}^{+}, i=1, \ldots, 5\right)$ and to NIS (vectors $\left.\hat{s}_{i}^{-}, i=1, \ldots, 5\right)$ are presented in Tables $\mathrm{V}$ and IV.

TABLE V

SIMILARITY VECTORS $\hat{s}_{i}^{+}=\left[s_{i 1}^{+}, \ldots, s_{i 5}^{+}\right]$OF THE ALTERNATIVES TO PIS REPRESENTED BY $V^{+}=[0,0,1,1,0]$

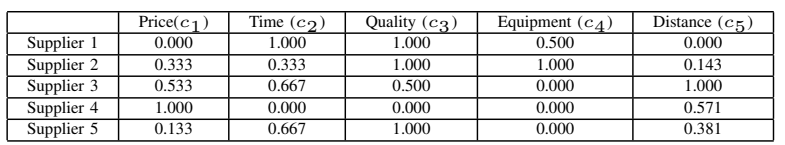

TABLE VI

SiMILARITY VECTORS $\hat{s}_{i}^{-}=\left[s_{i 1}^{-}, \ldots, s_{i 5}^{-}\right]$OF THE ALTERNATIVES TO NIS REPRESENTED BY $V^{-}=[1,1,0,0,1]$

\begin{tabular}{|l|c|c|c|c|c|}
\hline & Price $\left(c_{1}\right)$ & Time $\left(c_{2}\right)$ & Quality $\left(c_{3}\right)$ & Equipment $\left(c_{4}\right)$ & Distance $\left(c_{5}\right)$ \\
\hline Supplier 1 & 1.000 & 0.000 & 0.000 & 0.500 & 1.000 \\
\hline Supplier 2 & 0.667 & 0.667 & 0.000 & 0.000 & 0.857 \\
\hline Supplier 3 & 0.467 & 0.333 & 0.500 & 1.000 & 0.000 \\
\hline Supplier 4 & 1.000 & 0.000 & 0.000 & 1.000 & 0.429 \\
\hline Supplier 5 & 0.867 & 0.333 & 0.000 & 1.000 & 0.619 \\
\hline
\end{tabular}

We used Regular Increasing Monotone (RIM) type of quantifier guided aggregation in order to emphasize importance of getting high ratings from at least some criteria (or to be more precise to express in how many criteria we need to find high similarity with the respective ideal to consider the whole alternative similar to the ideal). We decided to use the exponential function $Q(x)=x^{\alpha}$ as our monotonic function. This choice here is simply based on the fact that it is the most commonly used quantifier function in the literature. Next we need to set up linguistic requirement for suppliers similarity towards PIS and NIS.

We will be considering three different cases representing different types of decision-makers:

\section{Careful decision-maker}

This decision-maker is rather pessimistic. To consider an alternative to be similar to PIS he/she requires high similarity in (at least) 'many' criteria between the given alternative and PIS. Note, that it is not specified in which criteria the similarity needs to be found. On the other hand similarity with NIS in (at least) 'few' criteria is considered enough by this decision-maker to consider the alternative similar to NIS (See Table I for linguistic evaluations). In other words this decision maker requires more strong evidence of high qualities of the given alternative to consider it good (similar to PIS), while some evidence of its badness is enough to consider it bad (similar to NIS). Such a behavior could be considered close to risk avoidance. This setup means, that 'many' will be represented by the following OWA weights for the calculation of the overall similarity to PIS:

$$
w^{+}=[0.04,0.12,0.2,0.28,0.36]
$$

derived using the equation (7) and the value of $\alpha=$ 2. The OWA weights used for the calculation of the overall similarity to NIS are calculated using (8) and the value of $\alpha=0.1$ :

$$
w^{-}=[0.8513,0.0611,0.0378,0.0277,0.0221] .
$$

Using these weights we can next calculate similarities of each alternative to positive ideal solution and to negative ideal solution. These can be found in Table VII. Similarly on the fourth column relative closeness values have been computed.

Based on the relative closeness values we get the ordering of suppliers to be $2 \succ 3 \succ 1 \succ 5 \succ 4$ meaning best choice of a supplier would be supplier 2 , the second best choice is supplier 3 etc.

TABLE VII

SIMILARITIES OF THE SUPPLIERS TO PIS AND NIS AND THE VALUES OF RELATIVE CLOSENESS OF THE SUPPLIERS TO PIS - THE CASE OF A CAREFUL (RISK-AVOIDING) DECISION-MAKER

\begin{tabular}{|c|c|c|c|}
\hline Attribute & $s^{+}$ & $s^{-}$ & RC \\
\hline Supplier 1 & 0.26 & 0.93 & 0.22 \\
\hline Supplier 2 & 0.37 & 0.80 & 0.32 \\
\hline Supplier 3 & 0.37 & 0.91 & 0.29 \\
\hline Supplier 4 & 0.11 & 0.96 & 0.10 \\
\hline Supplier 5 & 0.23 & 0.94 & 0.20 \\
\hline
\end{tabular}

\section{Optimistic decision-maker}

This decision-maker is much more willing to evaluate an alternative as good (similar to PIS) when its performance is similar with the performance of PIS in (at least) 'few' criteria. On the other hand to consider an alternative to be bad (similar to NIS) it would have to be similar to NIS in (at least) 'many' criteria. This approach can be considered close to risk-seeking. In this case:

$$
\begin{gathered}
w^{+}=[0.8513,0.0611,0.0378,0.0277,0.0221], \\
w^{-}=[0.04,0.12,0.2,0.28,0.36]
\end{gathered}
$$

The respective results can be found in Table VIII. We can see that in this more benevolent approach the relative closeness of all the alternatives to PIS is much larger than in the case of the careful decisionmaker. The suggested ordering of suppliers in this case would be $2 \succ 1 \succ 3 \succ 5 \succ 4$. We can see that while the most promising supplier remained the same, the runner up has changed from supplier 3 to 
TABLE VIII

SIMILARITIES OF THE SUPPLIERS TO PIS AND NIS AND THE VALUES OF RELATIVE CLOSENESS OF THE SUPPLIERS TO PIS - THE CASE OF AN OPTIMISTIC (RISK-SEEKING) DECISION-MAKER

\begin{tabular}{|c|c|c|c|}
\hline Attribute & $s^{+}$ & $s^{-}$ & RC \\
\hline Supplier 1 & 0.93 & 0.26 & 0.78 \\
\hline Supplier 2 & 0.94 & 0.25 & 0.79 \\
\hline Supplier 3 & 0.93 & 0.29 & 0.76 \\
\hline Supplier 4 & 0.89 & 0.48 & 0.65 \\
\hline Supplier 5 & 0.91 & 0.36 & 0.72 \\
\hline
\end{tabular}

supplier 1. This is the result of the best performance of supplier 1 in the two of the criteria. This ranking is more focused on the potential of the suppliers.

\section{Ignorant (risk-indifferent) decision-maker}

This decision-maker treats the similarity to PIS and to NIS in an identical way - for the alternative to be considered similar to PIS or to NIS its performance has to be similar with the given ideal in (at least) 'many' criteria. This approach is the closest to the original TOPSIS as it calculates the similarity to PIS and NIS in the same way. In this case

$$
w^{+}=w^{-}=[0.04,0.12,0.2,0.28,0.36]
$$

TABLE IX

SIMILARITIES OF THE SUPPLIERS TO PIS AND NIS AND THE VALUES OF RELATIVE CLOSENESS OF THE SUPPLIERS TO PIS - THE CASE OF AN IGNORANT (RISK-INDIFFERENT) DECISION-MAKER

\begin{tabular}{|c|c|c|c|}
\hline Attribute & $s^{+}$ & $s^{-}$ & $\mathrm{RC}$ \\
\hline Supplier 1 & 0.26 & 0.26 & 0.50 \\
\hline Supplier 2 & 0.37 & 0.25 & 0.60 \\
\hline Supplier 3 & 0.37 & 0.29 & 0.56 \\
\hline Supplier 4 & 0.11 & 0.48 & 0.18 \\
\hline Supplier 5 & 0.23 & 0.36 & 0.39 \\
\hline
\end{tabular}

The respective results can be found in Table IX. We can see that in this approach that treats both similarities to PIS and NIS in the same way we are getting the same final ordering as with the careful decision-maker. The suggested ordering of suppliers in this case would be $2 \succ 3 \succ 1 \succ 5 \succ 4$. The overall relative closeness to PIS is much larger for all the alternative than with the careful decisionmaker, but not as large as with the pessimistic one. This is due to the fact that the similarities of the alternatives to NIS are much smaller than with the careful decision-maker. The reason for this being that the ignorant/indifferent decision-maker requires the performance in 'many' criteria to be similar with the performance of NIS for the alternative to be considered similar to NIS and thus to lower the respective value of $R C_{i}$.

Obviously, there are many other possible choices of linguistic quantifications for the definition of overall similarity with PIS and NIS. In this paper we will focus on just these three.

We have, however, examined whether internal aggregations for all the combinations of linguistic quantifiers are different from each other by using Friedman's test. This is inline with [8] who studied aggregation of multiple experts with different OWA weights. The hypothesis in this case is

$\begin{aligned} H_{0}: & \text { The } 49 \text { rankings (combinations from } \\ & \alpha_{1}=0,0.1,0.5,1,2,10,1000 \text { and } \alpha_{2}= \\ & 0,0.1,0.5,1,2,10,1000) \text { of five alternatives are the } \\ & \text { same. } \\ H_{1} \text { : } & \text { At least two rankings are different }\end{aligned}$

TABLE X

FRIEDMAN TEST RESULT

\begin{tabular}{|c|c|}
\hline$\chi$ & 55.58 \\
\hline $\mathrm{df}$ & 4 \\
\hline $\mathrm{p}$ & $2.4584 e^{-11}$ \\
\hline
\end{tabular}

From the Friedman's test results we can conclude that the results are highly significant showing that by posing different linguistic requirements on similarity of supplier w.r.t. positive and negative ideal solutions it is possible to get significantly different ranking orders. These requirements need to reflect the needs and preferences (and potentially also the risk-attitude) of the decision-maker well, as for different linguistically quantified requirements we can get significantly different rankings of the alternatives.

\section{CONCLUSIONS}

In this paper similarity based TOPSIS with OWA operator is introduced. The advantage of using OWA operator in aggregation of similarities is that we are able to model such linguistic requirements as similarity should be high to at least some (at least half, most) criteria without needing to specify to which criteria. This changes decision making procedure clearly compared to situation where similarities/distances to particular criteria are needed to specify. Often in real world cases analysis requirements as 'at least some', 'at least half', 'most' are more suitable to practical problem at hand than the need to emphasize particular criteria. For this purpose similarity based TOPSIS with OWA operator is designed. Besides this it allows for the expression of preference/needs of the particular decision-maker with respect to what should be considered similar to PIS and NIS. From the presented examples it is clear that the same linguistic requirement may not be suitable for modeling requirements for both similarities toward PIS and NIS. For this purpose we allow the use of two different linguistic quantifiers reflecting the requirements of the decision-maker. We demonstrate the method by applying it to supplier selection problem for car manufacturing company in the context of three different types of decision-makers. Here we managed to show that different ranking orders can be gained which reflect of decision makers attitude towards situation at hand.

\section{REFERENCES}

[1] V. Traneva, S. Tranev, D. Mavrov, Interval-Valued Intuitionistic Fuzzy Decision-Making Method using Index Matrices and Application in Outsourcing, Annals of Computer Science and Information systems, 25, pp. 251-254, 2021, http://dx.doi.org/10.15439/2021F77 
[2] A. Karczmarczyk, J. Jankowski, J. Watrobski, Multi-criteria approach to viral marketing campaign planning in social networks, based on real networks, network samples and synthetic networks, Annals of Computer Science and Inofrmation systems, 18, pp. 663-673, 2019 http://dx.doi.org/10.15439/2019F199

[3] C.L. Hwang, K. Yoon, Multiple Attributes Decision Making Methods and Applications, 1980, Springer, Berlin, Heidelberg

[4] P. Luukka, M. Collan, Histogram ranking with generalized similarity-based TOPSIS applied to patent ranking, International Journal of Operational Research 25 (4), 2016, pp. 437-448, http://dx.doi.org/10.1504/IJOR.2016.075290

[5] P. Luukka, M. Collan, Bonferroni mean based similarity based TOPSIS, 2016 IEEE International Conference on Fuzzy Systems (FUZZ-IEEE), pp. 704-709, 2016, http://dx.doi.org/10.1109/FUZZ-IEEE.2016.7737756

[6] C. Bonferroni, Sulle medie multiple di potenze, Bollettino Matematica Italiana 5, 1950, pp. 267-270.

[7] R. R. Yager, On ordered weighted averaging aggregation operators in multi-criteria decision-making, Systems, Man and Cybernetics, IEEE Transactions, Vol. 18, 1988, pp. 183-190, 1988, http://dx.doi.org/10.1109/21.87068

[8] Y. Chen, K.W. Li, S. Liu, An OWA-TOPSIS method for multiple criteria decision analysis, Expert Systems with Applications, 38, pp. 5205-5211, 2011, http://dx.doi.org/10.1016/j.eswa.2010.10.039

[9] T. Wang, J. Liu, J. Li, C. Niu, An integrating OWA-TOPSIS framework in intuitionistic fuzzy settings for multiple attribute decision making, Computers \& Industrial Engineering, 98, pp. 185-194, 2016, http://dx.doi.org/10.1016/j.cie.2016.05.029

[10] F. Liu, Y-.F. Shang, L-.H. Pan, A modified TOPSIS method for obtaining the associated weights of the OWA-type operators, International journal of intelligent systems, 30, pp. 1101-1116, 2015, http://dx.doi.org/10.1002/int.21737

[11] B. Yusoff, J.M. Merigo, D.C. Hornero, Generalized OWA-TOPSIS model based on the concept of majority opinion for group decision making, FIM 2015, AISC 730, pp. 124-139, 2018.

[12] R. R. Yager, Quantifier Guided Aggregation using OWA Operators, International Journal of Intelligent Systems, Vol. 11, pp. 49-73, 1996, http://dx.doi.org/10.1002/(SICI)1098-111X(199601)11:1<49::AIDINT3>3.0.CO;2-Z

[13] Z. S. Xu, An Overview of Methods for Determining OWA Weights, International Journal of Intelligent Systems, Vol. 20, pp. 843-865, 2005, http://dx.doi.org/10.1002/int.20097 